\title{
A 3-D Differential Surface Admittance Operator for Lossy Dipole Antenna Analysis
}

\author{
Martijn Huynen ${ }^{1}$, Michiel Gossye, Daniël De Zutter, Fellow, IEEE, and Dries Vande Ginste, Senior Member, IEEE
}

\begin{abstract}
This paper presents a novel approach to simulate materials with arbitrary properties, in particular good conductors, in a boundary integral equation (BIE) context. The advocated differential surface admittance operator permits the replacement of the material by the background medium through the introduction of an equivalent surface current density. A formulation based on the eigenfunctions of the volume is constructed and successfully demonstrated through scattering at a conducting cylinder and through the analysis of a lossy dipole antenna.
\end{abstract}

Index Terms-dipole antenna, surface admittance, boundary integral equation.

\section{INTRODUCTION}

$\mathbf{R}$ ESEARCH into the influence of finite conductivity on antenna characteristics and performance has been going on for decades. An early technique modeled conductive, thin wire structures, such as cylindrical wires [1] or dipole antennas [2], by means of a postulated current distribution. Another approach uses the surface impedance concept. This impedance relates the external, tangential electric and magnetic field at the boundary of the object under study by a function that depends on the material's properties, as such eliminating the need to solve the internal field problem. Various methods exist [3] of which the Leontovich boundary condition is the most widely employed [4]. However, despite its versatility, the surface impedance formulation has a limited range of validity [5].

The same approach is adopted in the modeling of multiconductor transmission lines as well. Nonetheless, similar fundamental limitations apply, despite generalized impedance boundary conditions [6]. An alternative approach to calculate transmission line parameters for general 2-D cross sections was presented in [7]. Herein, the material properties of the various lines are taken into account by employing a differential surface admittance operator [8]. This operator enables the replacement of the material by the background medium through the introduction of a fictitious surface current density. This current density can then be calculated by conventional numerical techniques such as the method of moments (MoM).

In this letter, we extend the approach based on the Dirichletto Neumann-operator [8] from 2-D to 3-D configurations to efficiently study the influence of a dipole antenna's conductivity on its characteristics. Section II is devoted to the derivation of the differential surface admittance operator in 3-D and its specific form for a circular cylinder. In Section III, the novel formalism's validity and appositeness are illustrated with two numerical examples, i.e., the simulation of scattering at a

\footnotetext{
${ }^{1}$ IBCN/Electromagnetics Group, Department of Information Technology, Ghent University/iMinds, Technologiepark-Zwijnaarde 15, B-9052 Gent, Belgium. Contact e-mail: martijn.huynen@UGent.be
}

conducting cylinder and the analysis of a lossy dipole antenna. For the applications, emphasis is on thin-wire like bodies.

\section{THEORY}

Consider the time-harmonic ( $e^{j \omega t}$ dependence) electromagnetic fields in the geometry of Fig. 1a. The arbitrary volume $\mathcal{V}$ with boundary surface $\mathcal{S}$ is filled with a homogeneous, nonmagnetic material, characterized by its wavenumber $k$. The fields inside this region are denoted $\left(\mathbf{E}_{1}, \mathbf{H}_{1}\right)$. The fields on $\mathcal{S}$ are related by the Dirichlet-to-Neumann (DtN) operator $\mathcal{D}_{k}$ of the volume $\mathcal{V}$ [9]:

$$
\mathbf{u}_{n} \times \mathbf{H}_{1}=\mathcal{D}_{k} \mathbf{E}_{1}^{t},
$$

with the superscript $t$ denoting the tangential component of the corresponding field quantity and $\mathbf{u}_{n}$ the unit outward pointing normal to $\mathcal{S}$. The inner volume $\mathcal{V}$ is surrounded by another homogeneous volume $\mathcal{V}_{0}$ with different material parameters, i.e., a wavenumber $k_{0}$, but non-magnetic as well.

Now consider the similar case where the material of $\mathcal{V}$ is replaced by that of its surrounding volume $\mathcal{V}_{0}$, which corresponds to Fig. 1b. The fields inside the inner volume are now given by $(\mathbf{E}, \mathbf{H})$. These quantities are once more linked on the boundary by a DtN operator, viz.

$$
\mathbf{u}_{n} \times \mathbf{H}=\mathcal{D}_{k_{0}} \mathbf{E}^{t} .
$$

Furthermore, assume that the fields in the original situation in $\mathcal{V}_{0}$ are given by $\left(\mathbf{E}_{0}, \mathbf{H}_{0}\right)$. At this point, we want to replace the inner volume's material by that of the surrounding volume $\mathcal{V}_{0}$, effectively negating the discontinuity in material properties and as such arriving at the case of Fig. 1b. To preserve the field distribution outside the replaced volume, it suffices to impose a surface current density $\mathbf{J}_{s}$ on $\mathcal{S}$ related to the value of the tangential electric field on the boundary $\mathbf{E}_{0}^{t}$. Obviously, $\mathbf{E}_{0}^{t}$ must equal both $\mathbf{E}_{1}^{t}$ and $\mathbf{E}^{t}$ due to the continuity of the tangential electric field on $\mathcal{S}$. Hence, on this boundary surface $\mathcal{S}$, and only on $\mathcal{S}$, both internal tangential electric fields are identical. This is clearly not the case in the bulk of $\mathcal{V}$ where a fictitious field distribution is obtained in the equivalent situation of Fig. 1b. Combining (1) and (2) together with the boundary condition

$$
\mathbf{u}_{n} \times\left(\mathbf{H}_{1}-\mathbf{H}\right)=\mathbf{u}_{n} \times\left(\mathbf{H}_{0}-\mathbf{H}\right)=\mathbf{J}_{s},
$$

the equivalent surface current density is expressed by means of the differential surface admittance operator $\mathcal{Y}$ :

$$
\mathbf{J}_{s}=\mathcal{Y} \mathbf{E}_{1}^{t}=\left(\mathcal{D}_{k}-\mathcal{D}_{k_{0}}\right) \mathbf{E}_{1}^{t} .
$$

In the following, we derive an expression for this differential surface admittance operator based on the eigenmodes of the volume $\mathcal{V}$. 


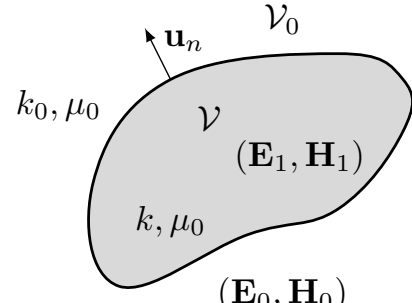

$\left(\mathbf{E}_{0}, \mathbf{H}_{0}\right)$

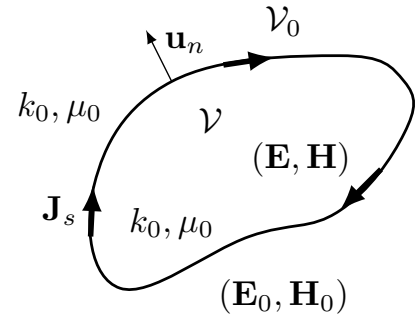

(b) Equivalent situation. (a) Original situation.

Fig. 1. Geometry of the problem. (a) A homogeneous volume $\mathcal{V}$ with boundary surface $\mathcal{S}$ is surrounded by another homogeneous volume $\mathcal{V}_{0}$. In (b), a surface current $\mathbf{J}_{s}$ is introduced on $\mathcal{S}$ and the inner material is replaced by that of the surrounding volume $\mathcal{V}_{0}$.

The electric fields inside $\mathcal{V}$ in both situations of Fig. 1 each satisfy the sourceless Helmholtz equation:

$$
\begin{aligned}
\nabla \times \nabla \times \mathbf{E}_{1}-k^{2} \mathbf{E}_{1} & =0, \\
\nabla \times \nabla \times \mathbf{E}-k_{0}^{2} \mathbf{E} & =0 .
\end{aligned}
$$

Subtracting (6) from (5) and defining $\mathcal{E}$ as $\mathbf{E}_{1}-\mathbf{E}$ shows that

$$
\nabla \times \nabla \times \mathcal{E}-k_{0}^{2} \mathcal{E}=\left(k^{2}-k_{0}^{2}\right) \mathbf{E}_{1},
$$

while $\mathbf{u}_{n} \times \mathcal{E}=0$. These relations imply that $\mathcal{E}$ can be expanded in electric solenoidal eigenvectors [10]

$$
\mathcal{E}=\sum_{l} a_{l} \mathbf{e}_{l}
$$

where the individual eigenmodes $\mathbf{e}_{l}$ satisfy

$$
\begin{aligned}
\nabla \times \nabla \times \mathbf{e}_{l}-k_{l}^{2} \mathbf{e}_{l} & =0, \\
\mathbf{u}_{n} \times \mathbf{e}_{l} & =0,
\end{aligned}
$$

with $k_{l}$ the wavenumber pertaining to the particular mode $\mathbf{e}_{l}$. Moreover, the eigenvectors are orthogonal. Consequently,

$$
\int_{\mathcal{V}} \mathbf{e}_{p} \cdot \mathbf{e}_{q}^{*} \mathrm{~d} V=\mathcal{N}_{p}^{2} \delta_{p q},
$$

with $\delta_{p q}$ the Kronecker delta and $\mathcal{N}_{p}^{2}$ a normalization factor.

Since both the eigenvectors and $\mathcal{E}$ satisfy the same boundary condition, we can safely apply the $\nabla \times \nabla \times$ operator to (8), which, by invoking (9), yields

$$
\nabla \times \nabla \times \mathcal{E}=\sum_{l} k_{l}^{2} a_{l} \mathbf{e}_{l}
$$

Substituting this expansion into (7), multiplying both sides with $\mathbf{e}_{m}^{*}$, and integrating over $\mathcal{V}$ leads to

$$
a_{m}\left(k_{m}^{2}-k_{0}^{2}\right) \mathcal{N}_{m}^{2}=\left(k^{2}-k_{0}^{2}\right)\left\langle\mathbf{E}_{1}, \mathbf{e}_{m}^{*}\right\rangle,
$$

by exploiting the orthogonality property (11). The volume integral in the scalar product $\left\langle\mathbf{E}_{1}, \mathbf{e}_{m}^{*}\right\rangle$ can be reduced to a surface integral confined to $\mathcal{S}$. To this end, we employ (9) to rewrite the scalar product and invoke the proper Green's theorem together with (10) to get

$$
k_{m}^{2}\left\langle\mathbf{E}_{1}, \mathbf{e}_{m}^{*}\right\rangle=k^{2}\left\langle\mathbf{E}_{1}, \mathbf{e}_{m}^{*}\right\rangle-\int_{\mathcal{S}}\left(\mathbf{u}_{n} \times \mathbf{E}_{1}\right) \cdot\left(\nabla \times \mathbf{e}_{m}^{*}\right) \mathrm{d} V .
$$

Now suppose we have normalized the eigenmodes such that

$$
\nabla \times \mathbf{e}_{m}=k_{m} \mathbf{h}_{m}
$$

and vice versa [10], with $\mathbf{h}_{m}$ the magnetic solenoidal eigenvectors. To emphasize that the tangential electric fields on $\mathcal{S}$ in both Fig. 1a and 1b are identical, we denote the tangential component of $\mathbf{E}_{1}$ on $\mathcal{S}$ as $\mathbf{E}_{1}^{t}$, as in (4). This yields the final expression for the coefficient $a_{m}$ :

$$
a_{m}=\frac{-k_{m}^{*}\left(k^{2}-k_{0}^{2}\right)}{\left(k_{m}^{2}-k_{0}^{2}\right)\left(k_{m}^{2}-k^{2}\right) \mathcal{N}_{m}^{2}} \int_{\mathcal{S}}\left(\mathbf{u}_{n} \times \mathbf{E}_{1}^{t}\right) \cdot \mathbf{h}_{m}^{*} \mathrm{~d} S .
$$

To conclude this derivation, we apply the curl to (8):

$$
\nabla \times \mathcal{E}=-j \omega \mu_{0}\left(\mathbf{H}_{1}-\mathbf{H}\right)=\sum_{l} k_{l} a_{l} \mathbf{h}_{l} .
$$

Employing (3) finally leads to an expression for the surface current density:

$$
\mathbf{J}_{s}=\mathcal{Y} \mathbf{E}_{1}^{t}=-\frac{1}{j \omega \mu_{0}} \sum_{l} k_{l} a_{l}\left(\mathbf{u}_{n} \times \mathbf{h}_{l}\right),
$$

which can be rewritten, by means of the contrast parameter $\eta=\left(k^{2}-k_{0}^{2}\right) / j \omega \mu_{0}=\sigma_{0}-\sigma+j \omega\left(\epsilon_{0}-\epsilon\right)$, as

$$
\mathbf{J}_{s}=\eta \sum_{l}\left[\frac{\left|k_{l}\right|^{2} \int_{\mathcal{S}}\left(\mathbf{u}_{n} \times \mathbf{E}_{1}^{t}\right) \cdot \mathbf{h}_{l}^{*} \mathrm{~d} S}{\left(k_{l}^{2}-k^{2}\right)\left(k_{l}^{2}-k_{0}^{2}\right) \mathcal{N}_{l}^{2}}\right]\left(\mathbf{u}_{n} \times \mathbf{h}_{l}\right) .
$$

\section{A. Circular cylinder}

The above form of the differential surface admittance operator $\mathcal{Y}$ requires the (magnetic) eigenvectors of the volume $\mathcal{V}$. Due to its omnipresence in antenna applications, we demonstrate the novel technique for the circular cylinder, whose eigenvectors and eigenvalues are easily found in literature. Note that for arbitrary shaped volumes, analytical solutions are generally not available and have to be constructed numerically.

The eigenvectors of a cylinder consist of two classes, viz. transversal electric (TE) and transversal magnetic (TM) modes. For a cylinder with radius $a$, height $L$ along the $z$-axis and its center at the origin, the TE eigenvectors and their corresponding wavenumbers are

$$
\begin{aligned}
\mathbf{h}_{n m p}^{\mathrm{TE}} & =\frac{-p \pi}{L}\left[\lambda_{n m} J_{n}^{\prime}\left(\lambda_{n m} \rho\right) \mathbf{u}_{\rho}+\frac{j n}{\rho} J_{n}\left(\lambda_{n m} \rho\right) \mathbf{u}_{\phi}\right] \\
& \cdot e^{j n \phi} \cos \left(\frac{p \pi}{L} z\right)-\lambda_{n m}^{2} J_{n}\left(\lambda_{n m} \rho\right) e^{j n \phi} \sin \left(\frac{p \pi}{L}\right) \mathbf{u}_{z}, \\
k_{n m p}^{2} & =\lambda_{n m}^{2}+\left(\frac{p \pi}{L}\right)^{2}=\left(\frac{y_{n m}}{a}\right)^{2}+\left(\frac{p \pi}{L}\right)^{2},
\end{aligned}
$$

with $J_{n}(x)$ the $n$-th order Bessel function and $y_{n m}$ the $m$-th zero of $J_{n}^{\prime}(x)$ [10]. Note that the index $l$ in (19) is in fact a triple index $\{n, m, p\}$ with $n \in[-N, N], m \in[1, M]$ and $p \in[0, P]$. Theoretically, $\{N, M, P\}$ are infinite but in computational experiments, they obviously have to be limited.

The TM modes, on the other hand, are defined by

$$
\begin{aligned}
\mathbf{h}_{n m p}^{\mathrm{TM}} & =k_{n m p}\left[\frac{j n}{\rho} J_{n}\left(\lambda_{n m} \rho\right) \mathbf{u}_{\rho}-\lambda_{n m} J_{n}^{\prime}\left(\lambda_{n m} \rho\right) \mathbf{u}_{\phi}\right] \\
& \cdot e^{j n \phi} \sin \left(\frac{p \pi}{L} z\right), \\
k_{n m p}^{2} & =\lambda_{n m}^{2}+\left(\frac{p \pi}{L}\right)^{2}=\left(\frac{x_{n m}}{a}\right)^{2}+\left(\frac{p \pi}{L}\right)^{2},
\end{aligned}
$$

with $x_{n m}$ the $m$-th zero of $J_{n}(x)$. Plugging these two sets of eigenvectors into (19) yields the differential surface admittance operator for the cylinder. 
What remains is to solve the exterior problem of the situation in Fig. 1b, also taking (4) into account. Due to its particular nature, this equivalent situation is ideally suited to be solved by a boundary integral equation (BIE) method such as the method of moments (MoM). In order to preserve the curved nature of the cylinder and facilitate the discretization of the $\mathcal{Y}$-operator, the surface is divided into curved rectangles on the side and conventional, flat triangles on the end caps. Both the surface current density and the tangential electric field on the boundary are projected on piecewise linear basis functions, i.e., generalized rooftops on the curved rectangles and RWGs on the triangles. This choice is permitted as, in contrast to, e.g., PMCHWT formulations, no derivatives of the tangential electric field are needed. For the BIE-MoM, Galerkin testing results in the matrix equation:

$$
\overline{\bar{G}} \cdot \mathbf{V}=\overline{\bar{Z}} \cdot \mathbf{I}+\mathbf{V}_{\text {inc }},
$$

where vectors $\mathbf{I}$ and $\mathbf{V}$ collect the unknown expansion coefficients for $\mathbf{J}_{s}$ and the tangential electric field, respectively, $\overline{\bar{G}}$ is the pertinent Gram matrix, $\overline{\bar{Z}}$ the discretized BIE-MoM operator and $\mathbf{V}_{\text {inc }}$ depends on the incident field.

Applying the same basis functions and testing strategy to (19), results in a second matrix equation

$$
\overline{\bar{G}} \cdot \mathbf{I}=\overline{\bar{Y}} \cdot \mathbf{V},
$$

with $\overline{\bar{Y}}$ the discretized version of the $\mathcal{Y}$-operator. Combining (24) and (25) finally yields the solution for the differential surface current density and the electric field.

\section{EXAMPLES}

\section{A. Validation example}

To validate the novel method, scattering at a single, homogeneous cylinder is considered for different materials. A reference solution is obtained from an in-house, all-purpose BIE-MoM solver. At $1 \mathrm{GHz}$, a cylinder with radius $r=\lambda / 3$ and height $2 \lambda$ is illuminated by a plane wave. The dimensions of the edges in the surface mesh are of the order $\lambda / 12$. Impinging at $\phi=0$ in the horizontal plane, i.e., in the plane perpendicular to the axis, the plane wave is TE-polarized, viz. its magnetic field is aligned with the axis of the cylinder. Three different materials are studied: a low contrast dielectric with $\epsilon_{r}=4$, a high contrast dielectric with $\epsilon_{r}=100$ and copper with a conductivity of $\sigma=5.8 \cdot 10^{7} \mathrm{~S} / \mathrm{m}$. Radar cross sections (RCSs) in the horizontal plane, halfway the cylinder's length are shown in Fig. 2 (only half the range is plotted due to symmetry). The solid lines represent the reference solution while the various markers demonstrate the solutions obtained by means of the differential surface admittance operator with the cut-off values of the eigenmodes at $\{N, M, P\}=\{15,200,25\}$ for the low contrast dielectric, $\{50,200,15\}$ for the cylinder with $\epsilon_{r}=100$ and $\{15,600,25\}$ for the copper filled cylinder.

As is evident from Fig. 2, excellent agreement between the reference solution and the novel formalism is found. The total rms error stays well below $1 \%$ for all examples, validating the accuracy of the differential surface admittance operator. The remaining, minor deviations can be attributed to the mesh

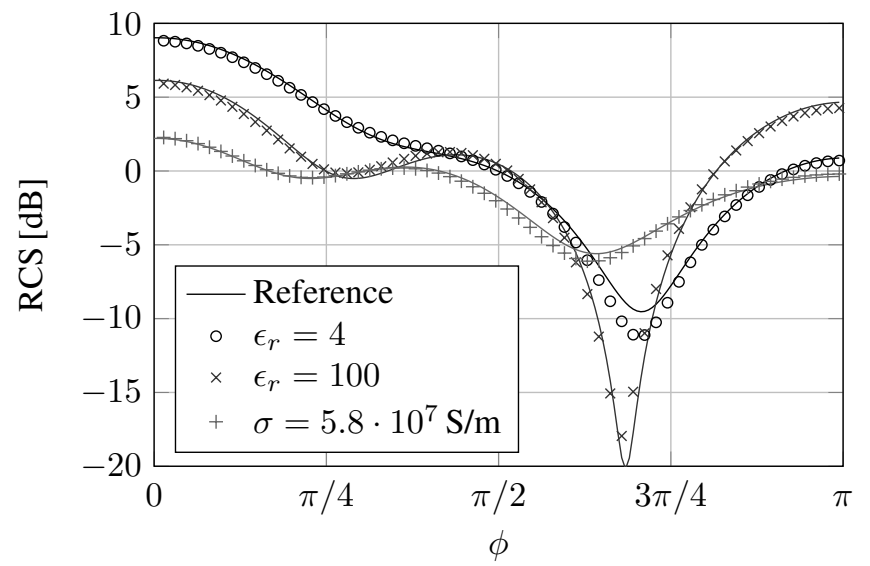

Fig. 2. Radar cross sections in the horizontal plane for a cylinder with radius $\lambda / 3$ and height $2 \lambda$ illuminated by a $1 \mathrm{GHz}$, TE-polarized plane wave impinging at $\phi=0$. Reference results for the simulated materials, i.e., a low contrast dielectric, a high contrast dielectric and copper, are obtained using in-house BIE-MoM software.

detail in both solvers, the finite number of eigenmodes in the novel method and rounding errors. Before turning our attention to an analysis of the influence of the numbers of eigenmodes, some preliminary remarks can already be made. For the dielectric cylinders, a higher number of eigenfunctions in the angular direction, i.e., a larger value of $N$, is utilized to fully capture the narrow peak in the RCS. For the copper cylinder, on the other hand, an increase in eigenmodes in the radial direction is required to capture the skin effect.

\section{B. Analysis of the discretized $\mathcal{Y}$-operator}

Before turning our attention to an application example, the behavior of the $\overline{\bar{Y}}$-matrix is explored in more detail. To this end, the evolution of three matrix elements for the copper cylinder with radius $r$ of Section III-A as a function of the relative skin depth $\delta / r=\sqrt{2 /(\omega \mu \sigma)} / r$ is examined: $y_{\text {hor }}$ and $y_{\text {ver }}$ both correspond to a diagonal element, the former for a horizontal edge and the latter for a vertical edge on the side of the cylinder. The third element $y_{\text {off }}$ is an off-diagonal matrix entry that represents the interaction between two vertical side edges on opposite sides of the cylinder. The real part (black lines) and imaginary part (gray lines) of these three quantities are shown in Fig. 3. The results demonstrate that the real parts are dominant and constant for large relative skin depths while the imaginary parts gain in importance as the skin depth decreases. For very small skin depths, only the diagonal elements remain significant and become proportional to the classical point-wise surface admittance $Y_{s}=\sqrt{\sigma /(j \omega \mu)}$, indicated by the identical absolute value of their real and imaginary part.

Another important influence on the matrix elements is the number of eigenmodes that are taken into account. To illustrate this effect, the diagonal elements of the previous example, i.e., $y_{\text {side }}$ and $y_{\text {top }}$, are computed over the same frequency range for an increasing number of eigenmodes in the radial direction, i.e., for increasing $M . N$ and $P$ are set to 5 for this particular example but note that the analysis remains valid for other parameter values as well. The relative error is plotted in Fig. 4 where the reference solution is the one obtained 


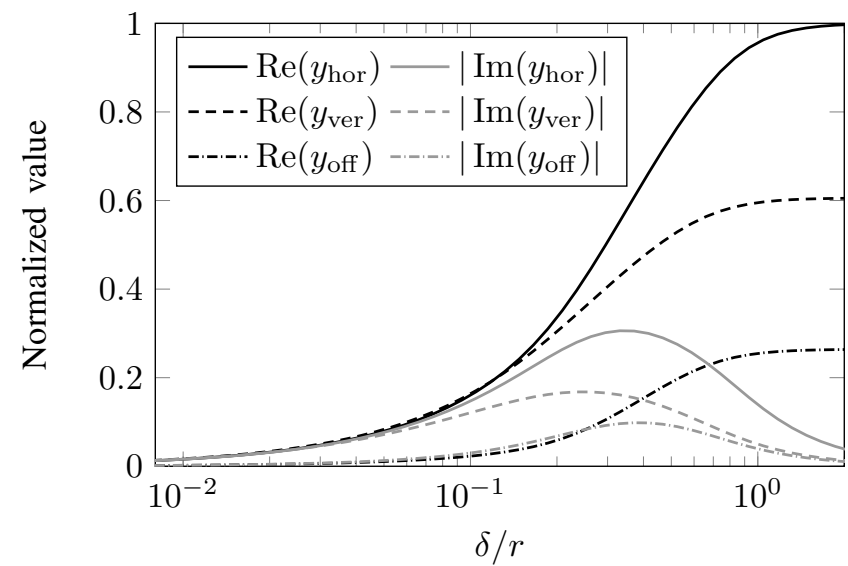

Fig. 3. Normalized real (black) and imaginary (gray) part of three selected $\overline{\bar{Y}}$-matrix elements as a function of the normalized skin depth.

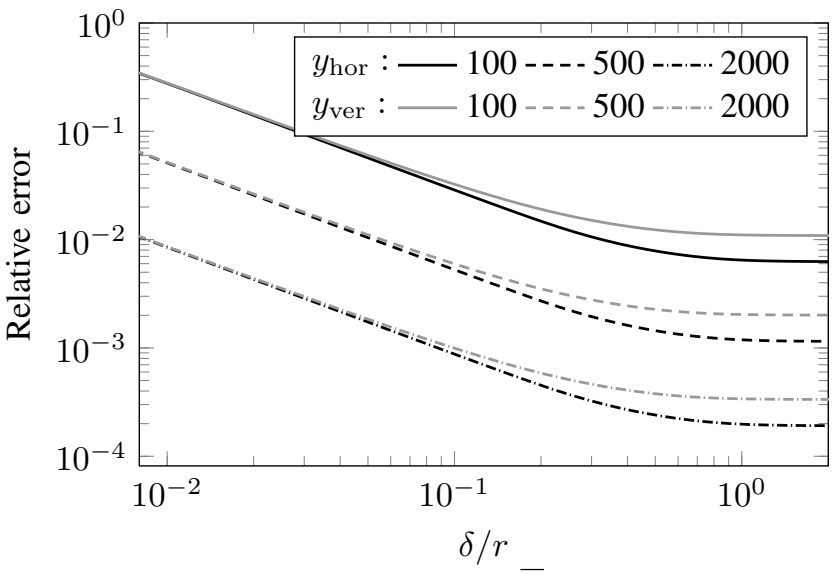

Fig. 4. Relative error of two distinct diagonal $\overline{\bar{Y}}$-matrix elements, i.e., $y_{\text {hor }}$ and $y_{\mathrm{ver}}$, as a function of normalized skin depth for the copper cylinder of Section III-A for various values of $M$. Values are defined with respect to the solution for $M=5000$.

for $M=5000$ as the absolute change in matrix entries was negligible for larger $M$. As is evident from the graph, a modest number of eigenmodes suffices for large skin depths while current crowding in the skin effect regime requires an increasing amount of eigenmodes to be fully captured.

\section{Application example}

Consider a dipole antenna in free space with a total length of $2 \lambda$ at $1 \mathrm{GHz}$. As the width of wire antennas is generally much smaller than their length (and the wavelength), the radius $r$ is set to $\lambda / 500$. The width of the gap between the two cylinders constituting the antenna, is set to $r / 50$. The structure is excited by an impinging TM-polarized plane wave for varying inclination angles $\theta$. The normalized open circuit voltage $V_{\text {open,norm }}$ over the dipole's gap, i.e., the potential difference between both cylinders, is numerically computed for increasing conductivities and displayed in Fig. 5. The voltage values are normalized with respect to the peak value of the solution for a PEC cylinder (denoted in Fig. 5 by the solid line). The range of eigenmodes in this example is given by $\{N, M, P\}=\{12,500,15\}$. Only half of the range of $\theta$ is plotted due to symmetry in the response characteristic. It is evident from the results that, with increasing conductivity,

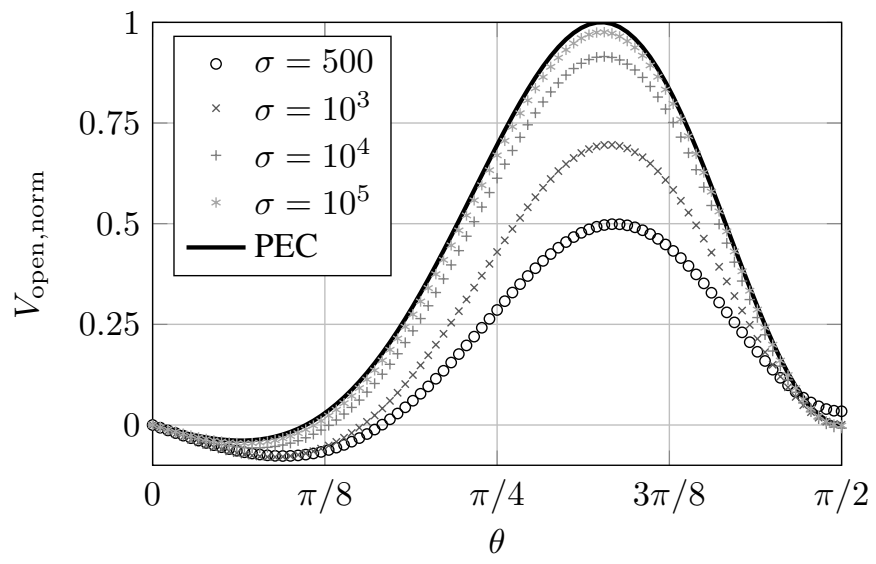

Fig. 5. Normalized open circuit voltage $V_{\text {open,norm of a dipole for a } 2 \lambda}$ dipole at $1 \mathrm{GHz}$ for various conductivities. Excitation by a TM-polarized plane wave at varying inclination angles.

the voltage response evolves towards that of the perfectly conducting cylinder, which is indeed to be expected. At the same time, however, it shows that even for a significant conductivity, the discrepancy between the idealized and lossy cylinder is not to be neglected.

\section{CONCLUSION}

In this contribution, a novel approach has been presented that employs the 3-D differential surface admittance operator to simulate materials of arbitrary properties in a BIE-MoM framework. An expression for this operator, based on the eigenfunctions of the volume, was derived and, more specifically, demonstrated for the circular cylinder. Thorough simulation and validation examples have shown its applicability to both scattering problems and antenna configurations.

\section{REFERENCES}

[1] E. Cassedy and J. Fainberg, "Back scattering cross sections of cylindrical wires of finite conductivity," IRE Trans. Antennas Propag., vol. 8, no. 1, pp. 1-7, Jan. 1960.

[2] R. King and T. Wu, "The imperfectly conducting cylindrical transmitting antenna," IEEE Trans. Antennas Propag., vol. 14, no. 5, pp. 524-534, Sep. 1966.

[3] L. Medgyesi-Mitschang and J. Putnam, "Integral equation formulations for imperfectly conducting scatterers," IEEE Trans. Antennas Propag., vol. 33, no. 2, pp. 206-214, Feb. 1985.

[4] R. D. Graglia, P. L. E. Uslenghi, R. Vitiello, and U. D'Elia, "Electromagnetic scattering for oblique incidence on impedance bodies of revolution," IEEE Trans. Antennas Propag., vol. 43, no. 1, pp. 11-26, Jan. 1995.

[5] D.-S. Wang, "Limits and validity of the impedance boundary condition on penetrable surfaces," IEEE Trans. Antennas Propag., vol. 35, no. 4, pp. 453-457, Apr. 1987.

[6] L. D. Rienzo, S. Yuferev, and N. Ida, "Computation of the impedance matrix of multiconductor transmission lines using high-order surface impedance boundary conditions," IEEE Trans. Electromagn. Compat., vol. 50, no. 4, pp. 974-984, Nov. 2008.

[7] T. Demeester and D. De Zutter, "Quasi-TM transmission line parameters of coupled lossy lines based on the Dirichlet to Neumann boundary operator," IEEE Trans. Microw. Theory Techn., vol. 56, no. 7, pp. 16491660, Jul. 2008

[8] D. De Zutter and L. Knockaert, "Skin effect modeling based on a differential surface admittance operator," IEEE Trans. Microw. Theory Techn., vol. 53, no. 8, pp. 2526-2538, Aug. 2005.

[9] H. Rogier, D. De Zutter, and L. Knockaert, "Two-dimensional transverse magnetic scattering using an exact surface admittance operator," Radio Science, vol. 42, no. 3, Jun. 2007.

[10] J. Van Bladel, Electromagnetic Fields. John Wiley \& Sons, 2007. 